\title{
Erratum to: Identification of clinical predictive factors of oxaliplatin-induced chronic peripheral neuropathy in colorectal cancer patients treated with adjuvant Folfox IV
}

\author{
Bruno Vincenzi • Anna Maria Frezza • Gaia Schiavon • \\ Chiara Spoto • Nicola Silvestris • Raffaele Addeo • \\ Vincenzo Catalano • Francesco Graziano • \\ Daniele Santini • Giuseppe Tonini
}

Published online: 25 January 2013

(C) Springer-Verlag Berlin Heidelberg 2013

\section{Erratum to: Support Care Cancer}

\section{DOI 10.1007/s00520-012-1667-5}

Unfortunately, the author Nicola Silvestris was omitted in the original version of the article. The missing author's name with affiliation and the correct order of authors are now corrected in this article.

The online version of the original article can be found at http://dx.doi.org/ $10.1007 / \mathrm{s} 00520-012-1667-5$.

B. Vincenzi $\cdot$ A. M. Frezza $\cdot$ C. Spoto $(\bowtie) \cdot$ D. Santini $\cdot$ G. Tonini Medical Oncology, Università Campus Bio-Medico,

Via Alvaro del Portillo 20000128 Rome, Italy

e-mail: c.spoto83@gmail.com

G. Schiavon

Department of Medical Oncology, Erasmus University Medical

Center-Daniel den Hoed Cancer Center, Postbus 2040,

3000 CA Rotterdam, Netherlands

N. Silvestris

Medical and Experimental Oncology Unit,

Cancer Institute "Giovanni Paolo II", Via Hahnemann,

1070125 Bari, Italy

R. Addeo

Ospedale San Giovanni Di Dio, Via Giovanni XXIII, 165,

80020 Frattaminore, Italy

V. Catalano

UOC Oncologia, A.O. "Ospedali Riuniti Marche Nord",

Presidio San Salvatore, Via Lombroso 1,

61100 Pesaro, Italy

F. Graziano

Dipartimento di Medicina Oncologica, Ospedale di Urbino,

Via Bonconte da Montefeltro,

61029 Urbino, Italy 